\title{
Weak convergence theorems of a hybrid algorithm in Hilbert spaces
}

Yan $\mathrm{HaO}^{*}$

\section{"Correspondence:}

zjhaoyan@aliyun.com

School of Mathematics, Physics and

Information Science, Zhejiang

Ocean University, Zhoushan,

316004, China

\begin{abstract}
In this paper, a hybrid algorithm is investigated for solving common solutions of a generalized equilibrium problem, a variational inequality, and fixed point problems of an asymptotically strict pseudocontraction. Weak convergence theorems are established in the framework of real Hilbert spaces.
\end{abstract}

Keywords: equilibrium problem; variational inequality; nonexpansive mapping; common solution

\section{Introduction}

Monotone variational inequalities recently have been investigated as an effective and powerful tool for studying a wide class of real world problems which arise in economics, finance, image reconstruction, ecology, transportation, and network; see [1-9] and the references therein. Monotone variational inequalities, which include many important problems in nonlinear analysis and optimization, such as the Nash equilibrium problem, complementarity problems, fixed point problems, saddle point problems, and game theory recently have been extensively studied based on projection methods. Many well-known problems can be studied by using methods which are iterative in their nature. As an example, in computer tomography with limited data, each piece of information implies the existence of a convex set in which the required solution lies. The problem of finding a point in the intersection of these convex subsets is then of crucial interest, and it cannot be usually solved directly. Therefore, an iterative algorithm must be used to approximate such a point. Krasnoselskii-Mann iteration, which is also known as a one-step iteration, is a classic algorithm to study fixed points of nonlinear operators. However, KrasnoselskiiMann iteration only enjoys weak convergence for nonexpansive mappings; see [10] and the references therein.

The purposes of this paper is to study common solutions of a generalized equilibrium problem, a variational inequality, and fixed point problems of an asymptotically strict pseudocontraction based on a hybrid algorithm. Weak convergence theorems are established in the framework of real Hilbert spaces. The organization of this paper is as follows. In Section 2, we provide some necessary preliminaries. In Section 3, a hybrid algorithm is introduced and the convergence analysis is given. Weak convergence theorems are established in a real Hilbert space.

○2014 Hao; licensee Springer. This is an Open Access article distributed under the terms of the Creative Commons Attribution License (http://creativecommons.org/licenses/by/2.0), which permits unrestricted use, distribution, and reproduction in any medium, provided the original work is properly cited. 


\section{Preliminaries}

From now on, we always assume that $H$ is a real Hilbert space with the inner product $\langle\cdot, \cdot\rangle$ and the norm $\|\cdot\|, C$ is a nonempty closed convex subset of $H$ and $P_{C}$ denotes the metric projection from $H$ onto $C$.

Let $A: C \rightarrow H$ be a mapping. Recall that $A$ is said to be monotone if

$$
\langle A x-A y, x-y\rangle \geq 0, \quad \forall x, y \in C .
$$

$A$ is said to be inverse-strongly monotone if there exists a constant $\alpha>0$ such that

$$
\langle A x-A y, x-y\rangle \geq \alpha\|A x-A y\|^{2}, \quad \forall x, y \in C .
$$

For such a case, we also call it an $\alpha$-inverse-strongly monotone mapping.

A set-valued mapping $T: H \rightarrow 2^{H}$ is said to be monotone if for all $x, y \in H, f \in T x$ and $g \in$ $T y$ imply $\langle x-y, f-g\rangle>0$. A monotone mapping $T: H \rightarrow 2^{H}$ is maximal if the graph $G(T)$ of $T$ is not properly contained in the graph of any other monotone mapping. It is known that a monotone mapping $T$ is maximal if and only if, for any $(x, f) \in H \times H,\langle x-y, f-g\rangle \geq 0$ for all $(y, g) \in G(T)$ implies $f \in T x$. Let $A$ be a monotone mapping of $C$ into $H$ and $N_{C} v$ be the normal cone to $C$ at $v \in C$, i.e.,

$$
N_{C} v=\{w \in H:\langle v-u, w\rangle \geq 0, \forall u \in C\}
$$

and define a mapping $T$ on $C$ by

$$
T v= \begin{cases}A v+N_{C} v, & v \in C, \\ \emptyset, & v \notin C .\end{cases}
$$

Then $T$ is maximal monotone and $0 \in T v$ if and only if $\langle A v, u-v\rangle \geq 0$ for all $u \in C$; see [6] and the references therein.

Recall that the classical variational inequality problem is to find $x \in C$ such that

$$
\langle A x, y-x\rangle \geq 0, \quad \forall y \in C .
$$

It is known that $x \in C$ is a solution to (2.1) if and only if $x$ is a fixed point of the mapping $P_{C}(I-\lambda A)$, where $\lambda>0$ is a constant and $I$ is the identity mapping. Projection methods recently have been studied for variational inequality (2.1); see [11-22] and the references therein.

Let $S: C \rightarrow C$ be a nonlinear mapping. In this paper, we use $F(S)$ to denote the fixed point set of $S$. Recall that $S$ is said to be nonexpansive if

$$
\|S x-S y\| \leq\|x-y\|, \quad \forall x, y \in C .
$$

$S$ is said to be asymptotically nonexpansive if there exists a sequence $\left\{k_{n}\right\} \subset[1, \infty)$ with $\lim _{n \rightarrow \infty} k_{n}=1$ such that

$$
\|S x-S y\| \leq k_{n}\|x-y\|, \quad \forall x, y \in C .
$$


$S$ is said to be $\kappa$-strictly pseudocontractive if there exists a constant $k \in[0,1)$ such that

$$
\|S x-S y\|^{2} \leq\|x-y\|^{2}+\kappa\|(x-S x)-(y-S y)\|^{2}, \quad \forall x, y \in C .
$$

The class of strict pseudocontractions was introduced by Browder and Petryshyn [23]. It is clear that every nonexpansive mapping is a 0 -strict pseudocontraction.

$T$ is said to be an asymptotically $\kappa$-strict pseudocontraction if there exists a sequence $\left\{k_{n}\right\} \subset[1, \infty)$ with $\lim _{n \rightarrow \infty} k_{n}=1$ and a constant $\kappa \in[0,1)$ such that

$$
\left\|T^{n} x-T^{n} y\right\|^{2} \leq k_{n}\|x-y\|^{2}+\kappa\left\|\left(I-T^{n}\right) x-\left(I-T^{n}\right) y\right\|^{2}, \quad \forall x, y \in C, n \geq 1 .
$$

The class of asymptotically strict pseudocontractions was introduced by Qihou [24]. It is clear that every asymptotically nonexpansive mapping is an asymptotically 0 -strict pseudocontraction.

Let $F$ be a bifunction of $C \times C$ into $\mathbb{R}$, where $\mathbb{R}$ denotes the set of real numbers and $A$ : $C \rightarrow H$ is an inverse-strongly monotone mapping. In this paper, we consider the following generalized equilibrium problem:

$$
\text { Find } x \in C \text { such that } F(x, y)+\langle A x, y-x\rangle \geq 0, \quad \forall y \in C \text {. }
$$

In this paper, the set of such $x \in C$ is denoted by $\operatorname{EP}(F, A)$, i.e.,

$$
\mathrm{EP}(F, A)=\{x \in C: F(x, y)+\langle A x, y-x\rangle \geq 0, \forall y \in C\}
$$

To study the generalized equilibrium problem (2.2), we may assume that $F$ satisfies the following conditions:

(A1) $F(x, x)=0$ for all $x \in C$;

(A2) $F$ is monotone, i.e., $F(x, y)+F(y, x) \leq 0$ for all $x, y \in C$;

(A3) for each $x, y, z \in C$,

$$
\limsup _{t \downarrow 0} F(t z+(1-t) x, y) \leq F(x, y) ;
$$

(A4) for each $x \in C, y \mapsto F(x, y)$ is convex and lower semi-continuous.

If $A \equiv 0$, then the generalized equilibrium problem (2.2) is reduced to the following equilibrium problem:

$$
\text { Find } x \in C \text { such that } F(x, y) \geq 0, \quad \forall y \in C \text {. }
$$

In this paper, the set of such $x \in C$ is denoted by $\operatorname{EP}(F)$, i.e.,

$$
\mathrm{EP}(F)=\{x \in C: F(x, y) \geq 0, \forall y \in C\}
$$

If $F \equiv 0$, then the generalized equilibrium problem (2.2) is reduced to the classical variational inequality (2.1).

Recently, equilibrium problems (2.2) and (2.3) have been investigated by many authors; see [25-31] and the references therein. Motivated by the research going on in this direction, we study a hybrid algorithm for solving common solutions of variational inequality 
(2.1), generalized equilibrium problem (2.2), and fixed points of an asymptotically strict pseudocontraction. Possible computation errors are taken into account. Weak convergence theorems are established in the framework of real Hilbert spaces.

In order to prove our main results, we also need the following lemmas.

Lemma 2.1 [32] Let $C$ be a nonempty closed convex subset of $H$, and let $F: C \times C \rightarrow \mathbb{R}$ be a bifunction satisfying (A1)-(A4). Then, for any $r>0$ and $x \in H$, there exists $z \in C$ such that

$$
F(z, y)+\frac{1}{r}\langle y-z, z-x\rangle \geq 0, \quad \forall y \in C .
$$

Further, define

$$
T_{r} x=\left\{z \in C: F(z, y)+\frac{1}{r}\langle y-z, z-x\rangle \geq 0, \forall y \in C\right\}
$$

for all $r>0$ and $x \in H$. Then the following hold:

(a) $T_{r}$ is single-valued;

(b) $T_{r}$ is firmly nonexpansive, i.e., for any $x, y \in H$,

$$
\left\|T_{r} x-T_{r} y\right\|^{2} \leq\left\langle T_{r} x-T_{r} y, x-y\right\rangle
$$

(c) $F\left(T_{r}\right)=\mathrm{EP}(F)$;

(d) $\mathrm{EP}(F)$ is closed and convex.

Lemma 2.2 [24] Let $C$ be a nonempty closed convex subset of a Hilbert space $H$ and $S$ : $C \rightarrow C$ be an asymptotically strict pseudocontraction. Then $I-S$ is demi-closed, that is, if $\left\{x_{n}\right\}$ is a sequence in $C$ with $x_{n} \rightarrow x$ and $x_{n}-S x_{n} \rightarrow 0$, then $x \in F(S)$.

Lemma 2.3 [33] Let $H$ be a Hilbert space and $0<p \leq t_{n} \leq q<1$ for all $n \geq 1$. Suppose that $\left\{x_{n}\right\}$ and $\left\{y_{n}\right\}$ are sequences in $H$ such that

$$
\limsup _{n \rightarrow \infty}\left\|x_{n}\right\| \leq d, \quad \limsup _{n \rightarrow \infty}\left\|y_{n}\right\| \leq d
$$

and

$$
\lim _{n \rightarrow \infty}\left\|t_{n} x_{n}+\left(1-t_{n}\right) y_{n}\right\|=d
$$

hold for some $d \geq 0$. Then $\lim _{n \rightarrow \infty}\left\|x_{n}-y_{n}\right\|=0$.

Lemma 2.4 [34] Let $\left\{a_{n}\right\},\left\{b_{n}\right\}$, and $\left\{c_{n}\right\}$ be three nonnegative sequences satisfying the following condition:

$$
a_{n+1} \leq\left(1+b_{n}\right) a_{n}+c_{n}, \quad \forall n \geq n_{0},
$$

where $n_{0}$ is some nonnegative integer, $\sum_{n=1}^{\infty} b_{n}<\infty$ and $\sum_{n=1}^{\infty} c_{n}<\infty$. Then the limit $\lim _{n \rightarrow \infty} a_{n}$ exists. 


\section{Main results}

Theorem 3.1 Let $C$ be a nonempty closed convex subset of a real Hilbert space H. Let $F$ be a bifunction from $C \times C$ to $\mathbb{R}$ which satisfies (A1)-(A4). Let $A: C \rightarrow H$ be an $\alpha$-inversestrongly monotone mapping, and let $B: C \rightarrow H$ be a $\beta$-inverse-strongly monotone mapping. Let $S: C \rightarrow C$ be an asymptotically $\kappa$-strict pseudocontraction with the sequence $\left\{k_{n}\right\}$ such that $\sum_{n=1}^{\infty}\left(k_{n}-1\right)<\infty$. Assume that $\Omega=F(S) \cap \mathrm{VI}(C, B) \cap \operatorname{EP}(F, A)$ is not empty. Let $\left\{\alpha_{n}\right\}$, $\left\{\alpha_{n}^{\prime}\right\},\left\{\alpha_{n}^{\prime \prime}\right\}$, and $\left\{\beta_{n}\right\}$ be real number sequences in $(0,1)$. Let $\left\{r_{n}\right\}$ and $\left\{s_{n}\right\}$ be two positive real number sequences. Let $\left\{x_{n}\right\}$ be a sequence generated in the following process:

$$
\left\{\begin{array}{l}
x_{1} \in C, \\
F\left(z_{n}, z\right)+\left\langle A x_{n}, z-z_{n}\right\rangle+\frac{1}{r_{n}}\left\langle z-z_{n}, z_{n}-x_{n}\right\rangle \geq 0, \quad \forall z \in C, \\
y_{n}=P_{C}\left(z_{n}-s_{n} B z_{n}\right), \\
x_{n+1}=\alpha_{n} x_{n}+\alpha_{n}^{\prime}\left(\beta_{n} y_{n}+\left(1-\beta_{n}\right) S^{n} y_{n}\right)+\alpha_{n}^{\prime \prime} e_{n},
\end{array}\right.
$$

where $\left\{e_{n}\right\}$ is a bounded sequence in C. Assume that the control sequences satisfy the following restrictions:

(a) $\alpha_{n}+\alpha_{n}^{\prime}+\alpha_{n}^{\prime \prime}=1$

(b) $0<p \leq \alpha_{n} \leq q<1$ and $\sum_{n=1}^{\infty} \alpha_{n}^{\prime \prime}<\infty$;

(c) $0<\kappa<\beta_{n} \leq b<1$;

(d) $0<s \leq s_{n} \leq s^{\prime}<2 \beta$ and $0<r \leq r_{n} \leq r^{\prime}<2 \alpha$,

where $p, q, b, s, s^{\prime}, r, r^{\prime}$ are real constants. Then $\left\{x_{n}\right\}$ converges weakly to some point in $\Omega$.

Proof First, we show that the sequences $\left\{x_{n}\right\},\left\{y_{n}\right\}$, and $\left\{z_{n}\right\}$ are bounded. Let $p \in \Omega$ be fixed arbitrarily. For any $x, y \in C$, we see that

$$
\begin{aligned}
\| & \left(I-r_{n} A\right) x-\left(I-r_{n} A\right) y \|^{2} \\
& =\left\|(x-y)-r_{n}(A x-A y)\right\|^{2} \\
& =\|x-y\|^{2}-2 r_{n}\langle x-y, A x-A y\rangle+r_{n}^{2}\|A x-A y\|^{2} \\
& \leq\|x-y\|^{2}-r_{n}\left(2 \alpha-r_{n}\right)\|A x-A y\|^{2} .
\end{aligned}
$$

Using the restriction (d), we see that $\left\|\left(I-r_{n} A\right) x-\left(I-r_{n} A\right) y\right\| \leq\|x-y\|$. This implies that $I-r_{n} A$ is nonexpansive. In the same way, we find that $I-s_{n} B$ is also nonexpansive. Using the restriction (c), we obtain that

$$
\begin{aligned}
&\left\|\beta_{n} y_{n}+\left(1-\beta_{n}\right) S^{n} y_{n}-p\right\|^{2} \\
&=\beta_{n}\left\|y_{n}-p\right\|^{2}+\left(1-\beta_{n}\right)\left\|S^{n} y_{n}-S^{n} p\right\|^{2} \\
& \quad-\beta_{n}\left(1-\beta_{n}\right)\left\|\left(y_{n}-p\right)-\left(S^{n} y_{n}-S^{n} p\right)\right\|^{2} \\
& \leq \quad \beta_{n}\left\|y_{n}-p\right\|^{2}+\left(1-\beta_{n}\right)\left(k_{n}\left\|y_{n}-p\right\|^{2}+\kappa\left\|\left(y_{n}-p\right)-\left(S^{n} y_{n}-S^{n} p\right)\right\|^{2}\right) \\
& \quad-\beta_{n}\left(1-\beta_{n}\right)\left\|\left(y_{n}-p\right)-\left(S^{n} y_{n}-S^{n} p\right)\right\|^{2} \\
&=k_{n}\left\|y_{n}-p\right\|^{2}-\left(1-\beta_{n}\right)\left(\beta_{n}-\kappa\right)\left\|\left(y_{n}-p\right)-\left(S^{n} y_{n}-S^{n} p\right)\right\|^{2} \\
& \leq k_{n}\left\|y_{n}-p\right\|^{2} .
\end{aligned}
$$


It follows that

$$
\begin{aligned}
\left\|x_{n+1}-p\right\|^{2} & \leq \alpha_{n}\left\|x_{n}-p\right\|^{2}+\alpha_{n}^{\prime}\left\|\beta_{n} y_{n}+\left(1-\beta_{n}\right) S^{n} y_{n}-p\right\|^{2}+\alpha_{n}^{\prime \prime}\left\|e_{n}-p\right\|^{2} \\
& \leq \alpha_{n}\left\|x_{n}-p\right\|^{2}+\alpha_{n}^{\prime} k_{n}\left\|y_{n}-p\right\|^{2}+\alpha_{n}^{\prime \prime}\left\|e_{n}-p\right\|^{2} \\
& =\alpha_{n}\left\|x_{n}-p\right\|^{2}+\alpha_{n}^{\prime} k_{n}\left\|P_{C}\left(I-s_{n} B\right) z_{n}-p\right\|^{2}+\alpha_{n}^{\prime \prime}\left\|e_{n}-p\right\|^{2} \\
& \leq \alpha_{n}\left\|x_{n}-p\right\|^{2}+\alpha_{n}^{\prime} k_{n}\left\|T_{r_{n}}\left(I-r_{n} A\right) x_{n}-p\right\|^{2}+\alpha_{n}^{\prime \prime}\left\|e_{n}-p\right\|^{2} \\
& \leq k_{n}\left\|x_{n}-p\right\|^{2}+\alpha_{n}^{\prime \prime}\left\|e_{n}-p\right\|^{2} .
\end{aligned}
$$

This implies from Lemma 2.4 that $\lim _{n \rightarrow \infty}\left\|x_{n}-p\right\|$ exists. This shows that $\left\{x_{n}\right\}$ is bounded, so are $\left\{y_{n}\right\}$ and $\left\{z_{n}\right\}$. From (3.2), we have

$$
\begin{aligned}
\left\|x_{n+1}-p\right\|^{2} & \leq \alpha_{n}\left\|x_{n}-p\right\|^{2}+\alpha_{n}^{\prime} k_{n}\left\|y_{n}-p\right\|^{2}+\alpha_{n}^{\prime \prime}\left\|e_{n}-p\right\|^{2} \\
& \leq \alpha_{n}\left\|x_{n}-p\right\|^{2}+\alpha_{n}^{\prime} k_{n}\left\|\left(I-s_{n} B\right) z_{n}-p\right\|^{2}+\alpha_{n}^{\prime \prime}\left\|e_{n}-p\right\|^{2} \\
& \leq \alpha_{n}\left\|x_{n}-p\right\|^{2}+\alpha_{n}^{\prime} k_{n}\left(\left\|z_{n}-p\right\|^{2}-s_{n}\left(2 \beta-s_{n}\right)\left\|B z_{n}-B p\right\|^{2}\right)+\alpha_{n}^{\prime \prime}\left\|e_{n}-p\right\|^{2} \\
& \leq k_{n}\left\|x_{n}-p\right\|^{2}-s_{n} k_{n} \alpha_{n}^{\prime}\left(2 \beta-s_{n}\right)\left\|B z_{n}-B p\right\|^{2}+\alpha_{n}^{\prime \prime}\left\|e_{n}-p\right\|^{2} .
\end{aligned}
$$

It follows that

$$
s_{n} k_{n} \alpha_{n}^{\prime}\left(2 \beta-s_{n}\right)\left\|B z_{n}-B p\right\|^{2} \leq k_{n}\left\|x_{n}-p\right\|^{2}-\left\|x_{n+1}-p\right\|^{2}+\alpha_{n}^{\prime \prime}\left\|e_{n}-p\right\|^{2} .
$$

With the aid of the restrictions (b) and (d), we find that

$$
\lim _{n \rightarrow \infty}\left\|B z_{n}-B p\right\|=0
$$

Since $P_{C}$ is firmly nonexpansive, we have

$$
\begin{aligned}
\left\|y_{n}-p\right\|^{2}= & \left\|P_{C}\left(I-s_{n} B\right) z_{n}-P_{C}\left(I-s_{n} B\right) p\right\|^{2} \\
\leq & \left\langle\left(I-s_{n} B\right) z_{n}-\left(I-s_{n} B\right) p, y_{n}-p\right\rangle \\
= & \frac{1}{2}\left\{\left\|\left(I-s_{n} B\right) z_{n}-\left(I-s_{n} B\right) p\right\|^{2}+\left\|y_{n}-p\right\|^{2}\right. \\
& \left.-\left\|\left(I-s_{n} B\right) z_{n}-\left(I-s_{n} B\right) p-\left(y_{n}-p\right)\right\|^{2}\right\} \\
\leq & \frac{1}{2}\left\{\left\|z_{n}-p\right\|^{2}+\left\|y_{n}-p\right\|^{2}-\left\|z_{n}-y_{n}-s_{n}\left(B z_{n}-B p\right)\right\|^{2}\right\} \\
= & \frac{1}{2}\left\{\left\|z_{n}-p\right\|^{2}+\left\|y_{n}-p\right\|^{2}-\left\|z_{n}-y_{n}\right\|^{2}\right. \\
& \left.+2 s_{n}\left\langle z_{n}-y_{n}, B z_{n}-B p\right\rangle-s_{n}^{2}\left\|B z_{n}-B p\right\|^{2}\right\} \\
\leq & \frac{1}{2}\left\{\left\|x_{n}-p\right\|^{2}+\left\|y_{n}-p\right\|^{2}-\left\|z_{n}-y_{n}\right\|^{2}\right. \\
& \left.+2 s_{n}\left\langle z_{n}-y_{n}, B z_{n}-B p\right\rangle-s_{n}^{2}\left\|B z_{n}-B p\right\|^{2}\right\},
\end{aligned}
$$

which implies that

$$
\left\|y_{n}-p\right\|^{2} \leq\left\|x_{n}-p\right\|^{2}-\left\|z_{n}-y_{n}\right\|^{2}+2 s_{n}\left\|z_{n}-y_{n}\right\|\left\|B z_{n}-B p\right\| .
$$


Hence, we find from (3.2) that

$$
\begin{aligned}
\left\|x_{n+1}-p\right\|^{2} \leq & \alpha_{n}\left\|x_{n}-p\right\|^{2}+\alpha_{n}^{\prime} k_{n}\left\|y_{n}-p\right\|^{2}+\alpha_{n}^{\prime \prime}\left\|e_{n}-p\right\|^{2} \\
\leq & k_{n}\left\|x_{n}-p\right\|^{2}-\alpha_{n}^{\prime} k_{n}\left\|z_{n}-y_{n}\right\|^{2}+2 \alpha_{n}^{\prime} s_{n} k_{n}\left\|z_{n}-y_{n}\right\|\left\|B z_{n}-B p\right\| \\
& +\alpha_{n}^{\prime \prime}\left\|e_{n}-p\right\|^{2} .
\end{aligned}
$$

Therefore, we obtain that

$$
\begin{aligned}
\alpha_{n}^{\prime} k_{n}\left\|z_{n}-y_{n}\right\|^{2} \leq & k_{n}\left\|x_{n}-p\right\|^{2}-\left\|x_{n+1}-p\right\|^{2}+2 s_{n} k_{n}\left\|z_{n}-y_{n}\right\|\left\|B z_{n}-B p\right\| \\
& +\alpha_{n}^{\prime \prime}\left\|e_{n}-p\right\|^{2} .
\end{aligned}
$$

From the restrictions (b) and (d), we find from (3.3) that

$$
\lim _{n \rightarrow \infty}\left\|z_{n}-y_{n}\right\|=0
$$

It follows from (3.1) that

$$
\begin{aligned}
\left\|z_{n}-p\right\|^{2} & =\left\|T_{r_{n}}\left(I-r_{n} A\right) x_{n}-p\right\|^{2} \\
& \leq\left\|x_{n}-p\right\|^{2}-r_{n}\left(2 \alpha-r_{n}\right)\left\|A x_{n}-A p\right\|^{2} .
\end{aligned}
$$

Hence, we have

$$
\begin{aligned}
\left\|x_{n+1}-p\right\|^{2} & \leq \alpha_{n}\left\|x_{n}-p\right\|^{2}+\alpha_{n}^{\prime} k_{n}\left\|y_{n}-p\right\|^{2}+\alpha_{n}^{\prime \prime}\left\|e_{n}-p\right\|^{2} \\
& \leq \alpha_{n}\left\|x_{n}-p\right\|^{2}+\alpha_{n}^{\prime} k_{n}\left\|z_{n}-p\right\|^{2}+\alpha_{n}^{\prime \prime}\left\|e_{n}-p\right\|^{2} \\
& \leq k_{n}\left\|x_{n}-p\right\|^{2}-\alpha_{n}^{\prime} r_{n}\left(2 \alpha-r_{n}\right) k_{n}\left\|A x_{n}-A p\right\|^{2}+\alpha_{n}^{\prime \prime}\left\|e_{n}-p\right\|^{2} .
\end{aligned}
$$

This implies that

$$
\alpha_{n}^{\prime} r_{n}\left(2 \alpha-r_{n}\right) k_{n}\left\|A x_{n}-A p\right\|^{2} \leq k_{n}\left\|x_{n}-p\right\|^{2}-\left\|x_{n+1}-p\right\|^{2}+\alpha_{n}^{\prime \prime}\left\|e_{n}-p\right\|^{2} .
$$

Using the restrictions (b) and (d), we obtain that

$$
\lim _{n \rightarrow \infty}\left\|A x_{n}-A p\right\|=0
$$

Since $T_{r_{n}}$ is firmly nonexpansive, we find that

$$
\begin{aligned}
\left\|z_{n}-p\right\|^{2}= & \left\|T_{r_{n}}\left(I-r_{n} A\right) x_{n}-T_{r_{n}}\left(I-r_{n} A\right) p\right\|^{2} \\
\leq & \left\langle\left(I-r_{n} A\right) x_{n}-\left(I-r_{n} A\right) p, z_{n}-p\right\rangle \\
= & \frac{1}{2}\left(\left\|\left(I-r_{n} A\right) x_{n}-\left(I-r_{n} A\right) p\right\|^{2}+\left\|z_{n}-p\right\|^{2}\right. \\
& \left.-\left\|\left(I-r_{n} A\right) x_{n}-\left(I-r_{n} A\right) p-\left(z_{n}-p\right)\right\|^{2}\right) \\
\leq & \frac{1}{2}\left(\left\|x_{n}-p\right\|^{2}+\left\|z_{n}-p\right\|^{2}-\left\|x_{n}-z_{n}-r_{n}\left(A x_{n}-A p\right)\right\|^{2}\right)
\end{aligned}
$$




$$
\begin{aligned}
= & \frac{1}{2}\left(\left\|x_{n}-p\right\|^{2}+\left\|z_{n}-p\right\|^{2}-\left(\left\|x_{n}-z_{n}\right\|^{2}\right.\right. \\
& \left.\left.-2 r_{n}\left\langle x_{n}-z_{n}, A x_{n}-A p\right\rangle-r_{n}^{2}\left\|A x_{n}-A p\right\|^{2}\right)\right),
\end{aligned}
$$

which implies that

$$
\left\|z_{n}-p\right\|^{2} \leq\left\|x_{n}-p\right\|^{2}-\left\|x_{n}-z_{n}\right\|^{2}+2 r_{n}\left\|x_{n}-z_{n}\right\|\left\|A x_{n}-A p\right\| .
$$

It follows that

$$
\begin{aligned}
\left\|x_{n+1}-p\right\|^{2} \leq & \alpha_{n}\left\|x_{n}-p\right\|^{2}+\alpha_{n}^{\prime} k_{n}\left\|y_{n}-p\right\|^{2}+\alpha_{n}^{\prime \prime}\left\|e_{n}-p\right\|^{2} \\
\leq & \alpha_{n}\left\|x_{n}-p\right\|^{2}+\alpha_{n}^{\prime} k_{n}\left\|z_{n}-p\right\|^{2}+\alpha_{n}^{\prime \prime}\left\|e_{n}-p\right\|^{2} \\
\leq & k_{n}\left\|x_{n}-p\right\|^{2}-\alpha_{n}^{\prime} k_{n}\left\|x_{n}-z_{n}\right\|^{2}+2 r_{n} \alpha_{n}^{\prime} k_{n}\left\|x_{n}-z_{n}\right\|\left\|A x_{n}-A p\right\| \\
& +\alpha_{n}^{\prime \prime}\left\|e_{n}-p\right\|^{2},
\end{aligned}
$$

which yields that

$$
\begin{aligned}
\alpha_{n}^{\prime} k_{n}\left\|x_{n}-z_{n}\right\|^{2} \leq & k_{n}\left\|x_{n}-p\right\|^{2}-\left\|x_{n+1}-p\right\|^{2}+2 r_{n} \alpha_{n}^{\prime}\left\|x_{n}-z_{n}\right\|\left\|A x_{n}-A p\right\| \\
& +\alpha_{n}^{\prime \prime}\left\|e_{n}-p\right\|^{2} .
\end{aligned}
$$

Using the restrictions (b) and (d), we find from (3.5) that

$$
\lim _{n \rightarrow \infty}\left\|x_{n}-z_{n}\right\|=0
$$

It follows from (3.4) and (3.6) that

$$
\lim _{n \rightarrow \infty}\left\|x_{n}-y_{n}\right\|=0
$$

Since $\left\{x_{n}\right\}$ is bounded, we see that there exists a subsequence $\left\{x_{n_{i}}\right\}$ of $\left\{x_{n}\right\}$ which converges weakly to $\xi$. Let $T$ be a maximal monotone mapping defined by

$$
T x= \begin{cases}B x+N_{C} x, & x \in C, \\ \emptyset, & x \notin C .\end{cases}
$$

For any given $(x, y) \in \operatorname{Graph}(T)$, we have $y-B x \in N_{C} x$. Since $y_{n} \in C$, by the definition of $N_{C}$, we have $\left\langle x-y_{n}, y-B x\right\rangle \geq 0$. Since $y_{n}=P_{C}\left(I-s_{n} B\right) z_{n}$, we see that $\left\langle x-y_{n}, y_{n}-\left(I-s_{n} B\right) z_{n}\right\rangle \geq 0$ and hence

$$
\left\langle x-y_{n}, \frac{y_{n}-z_{n}}{s_{n}}+B z_{n}\right\rangle \geq 0 .
$$

It follows that

$$
\begin{aligned}
\left\langle x-y_{n_{i}}, y\right\rangle & \geq\left\langle x-y_{n_{i}}, B x\right\rangle \\
& \geq\left\langle x-y_{n_{i}}, B x\right\rangle-\left\langle x-y_{n_{i}}, \frac{y_{n_{i}}-z_{n_{i}}}{s_{n_{i}}}+B z_{n_{i}}\right\rangle
\end{aligned}
$$




$$
\begin{aligned}
& =\left\langle x-y_{n_{i}}, B x-B y_{n_{i}}\right\rangle+\left\langle x-y_{n_{i}}, B y_{n_{i}}-B z_{n_{i}}\right\rangle-\left\langle x-y_{n_{i}}, \frac{y_{n_{i}}-z_{n_{i}}}{s_{n_{i}}}\right\rangle \\
& \geq\left\langle x-y_{n_{i}}, B y_{n_{i}}-B z_{n_{i}}\right\rangle-\left\langle x-y_{n_{i}}, \frac{y_{n_{i}}-z_{n_{i}}}{s_{n_{i}}}\right\rangle .
\end{aligned}
$$

Since $y_{n_{i}}$ converges weakly to $\xi$ and $B$ is $\frac{1}{\beta}$-Lipschitz continuous, we see that $\langle x-\xi, y\rangle \geq 0$. Notice that $T$ is maximal monotone and hence $0 \in T \xi$. This shows that $\xi \in \operatorname{VI}(C, B)$. From (3.6), we see that $z_{n_{i}}$ converges weakly to $\xi$. It follows that

$$
F\left(z_{n}, z\right)+\left\langle A x_{n}, z-z_{n}\right\rangle+\frac{1}{r_{n}}\left\langle z-z_{n}, z_{n}-x_{n}\right\rangle \geq 0, \quad \forall z \in C .
$$

From condition (A2), we see that

$$
\left\langle A x_{n}, z-z_{n}\right\rangle+\frac{1}{r_{n}}\left\langle z-z_{n}, z_{n}-x_{n}\right\rangle \geq F\left(z, z_{n}\right), \quad \forall z \in C .
$$

Replacing $n$ by $n_{i}$, we arrive at

$$
\left\langle A x_{n_{i}}, z-z_{n_{i}}\right\rangle+\left\langle z-z_{n_{i}}, \frac{z_{n_{i}}-x_{n_{i}}}{r_{n_{i}}}\right\rangle \geq F\left(z, z_{n_{i}}\right), \quad \forall z \in C .
$$

For $t$ with $0<t \leq 1$ and $z \in C$, let $u_{t}=t z+(1-t) \xi$. Since $z \in C$ and $\xi \in C$, we have $u_{t} \in C$. In view of (3.8), we find that

$$
\begin{aligned}
\left\langle u_{t}-z_{n_{i}}, A u_{t}\right\rangle \geq & \left\langle u_{t}-z_{n_{i}}, A u_{t}\right\rangle-\left\langle A x_{n_{i}}, u_{t}-z_{n_{i}}\right\rangle-\left\langle u_{t}-z_{n_{i}}, \frac{z_{n_{i}}-x_{n_{i}}}{r_{n_{i}}}\right\rangle \\
& +F\left(u_{t}, z_{n_{i}}\right) \\
= & \left\langle u_{t}-z_{n_{i}}, A u_{t}-A z_{n_{i}}\right\rangle+\left\langle u_{t}-z_{n_{i}}, A z_{n_{i}}-A x_{n_{i}}\right\rangle \\
& -\left\langle u_{t}-z_{n_{i}}, \frac{z_{n_{i}}-x_{n_{i}}}{r_{n_{i}}}\right\rangle+F\left(u_{t}, z_{n_{i}}\right) .
\end{aligned}
$$

Using (3.6), we have $\lim _{i \rightarrow \infty} A z_{n_{i}}-A x_{n_{i}}=0$. Since $A$ is monotone, we see that $\left\langle u_{t}-\right.$ $\left.z_{n_{i}}, A u_{t}-A z_{n_{i}}\right\rangle \geq 0$. It follows from condition (A4) that

$$
\left\langle u_{t}-\xi, A u_{t}\right\rangle \geq F\left(u_{t}, \xi\right)
$$

Using conditions (A1) and (A4), we see from (3.9) that

$$
\begin{aligned}
0 & =F\left(u_{t}, u_{t}\right) \leq t F\left(u_{t}, z\right)+(1-t) F\left(u_{t}, \xi\right) \\
& \leq t F\left(u_{t}, z\right)+(1-t)\left\langle u_{t}-\xi, A u_{t}\right\rangle \\
& =t F\left(u_{t}, u\right)+(1-t) t\left\langle z-\xi, A u_{t}\right\rangle,
\end{aligned}
$$

which yields that

$$
F\left(u_{t}, z\right)+(1-t)\left\langle z-\xi, A u_{t}\right\rangle \geq 0
$$


Letting $t \rightarrow 0$, we find

$$
F(\xi, z)+\langle z-\xi, A \xi\rangle \geq 0
$$

which implies that $\xi \in \mathrm{EP}(F, A)$.

Now, we are in a position to show $\xi \in F(S)$. Since $\lim _{n \rightarrow \infty}\left\|x_{n}-p\right\|$ exists, we may assume that $\lim _{n \rightarrow \infty}\left\|x_{n}-p\right\|=d>0$. Put $\lambda_{n}=\beta_{n} y_{n}+\left(1-\beta_{n}\right) S^{n} y_{n}$. It follows from (3.2) that $\lim \sup _{n \rightarrow \infty}\left\|x_{n}-p+\alpha_{n}^{\prime \prime}\left(e_{n}-\lambda_{n}\right)\right\| \leq d$ and $\limsup _{n \rightarrow \infty}\left\|\lambda_{n}-p+\alpha_{n}^{\prime \prime}\left(e_{n}-\lambda_{n}\right)\right\| \leq d$. On the other hand, we have

$$
\lim _{n \rightarrow \infty}\left\|x_{n+1}-p\right\|=\lim _{n \rightarrow \infty}\left\|\alpha_{n}\left(\left(x_{n}-p\right)+\alpha_{n}^{\prime \prime}\left(e_{n}-\lambda_{n}\right)\right)+\left(1-\alpha_{n}\right)\left(\lambda_{n}-p+\alpha_{n}^{\prime \prime}\left(e_{n}-\lambda_{n}\right)\right)\right\|=d .
$$

Using Lemma 2.3, we obtain that $\lim _{n \rightarrow \infty}\left\|\lambda_{n}-x_{n}\right\|=0$. Note that

$$
S^{n} y_{n}-x_{n}=\frac{\lambda_{n}-x_{n}}{1-\beta_{n}}+\frac{\beta_{n}\left(x_{n}-y_{n}\right)}{1-\beta_{n}} .
$$

Hence, we have $\lim _{n \rightarrow \infty}\left\|S^{n} y_{n}-x_{n}\right\|=0$. Note that $\left\|S^{n} x_{n}-x_{n}\right\| \leq\left\|S^{n} x_{n}-S^{n} y_{n}\right\|+\| S^{n} y_{n}-$ $x_{n} \|$. Since $S$ is Lipschitz continuous, we have $\lim _{n \rightarrow \infty}\left\|S^{n} x_{n}-x_{n}\right\|=0$. Further, we find that $\lim _{n \rightarrow \infty}\left\|S x_{n}-x_{n}\right\|=0$. Using Lemma 2.2, we see that $\xi \in F(S)$. This proves that $\eta \in \Omega$.

Finally, we show that the sequence $\left\{x_{n}\right\}$ converges weakly to $\xi$. Assume that there exists another subsequence $\left\{x_{n_{j}}\right\}$ of $\left\{x_{n}\right\}$ such that $\left\{x_{n_{j}}\right\}$ converges weakly to $\eta$. In the same way, we find $\eta \in \Omega$. If $\eta \neq \xi$, we see from the Opial condition [35] that

$$
\begin{aligned}
\lim _{n \rightarrow \infty}\left\|x_{n}-\xi\right\| & =\liminf _{i \rightarrow \infty}\left\|x_{n_{i}}-\xi\right\|<\liminf _{i \rightarrow \infty}\left\|x_{n_{i}}-\eta\right\| \\
& =\liminf _{n \rightarrow \infty}\left\|x_{n}-\eta\right\|=\liminf _{j \rightarrow \infty}\left\|x_{n_{j}}-\eta\right\| \\
& <\liminf _{j \rightarrow \infty}\left\|x_{n_{j}}-\xi\right\|=\lim _{n \rightarrow \infty}\left\|x_{n}-\xi\right\| .
\end{aligned}
$$

This derives a contradiction. Hence, we have $\eta=\xi$. This implies that $x_{n} \rightarrow \xi \in \Omega$. This completes the proof.

Remark 3.2 The key of the weak convergence of the algorithm is due to the fact that $A$ is inverse-strongly monotone, which yields that $I-r_{n} A$ is nonexpansive. The nonexpansivity of the mapping $I-r_{n} A$ plays an important role in this theorem. Therefore, it is of interest to relax the monotonicity of $A$ such that the algorithm is still weakly convergent.

Next, we give some subresults of Theorem 3.1. If $S$ is asymptotically nonexpansive, we find the following result.

Corollary 3.3 Let $C$ be a nonempty closed convex subset of a real Hilbert space H. Let $F$ be a bifunction from $C \times C$ to $\mathbb{R}$ which satisfies (A1)-(A4). Let $A: C \rightarrow H$ be an $\alpha$-inversestrongly monotone mapping, and let $B: C \rightarrow H$ be a $\beta$-inverse-strongly monotone mapping. Let $S: C \rightarrow C$ be an asymptotically nonexpansive mapping with the sequence $\left\{k_{n}\right\}$ such that $\sum_{n=1}^{\infty}\left(k_{n}-1\right)<\infty$. Assume that $\Omega=F(S) \cap \operatorname{VI}(C, B) \cap \operatorname{EP}(F, A)$ is not empty. Let $\left\{\alpha_{n}\right\},\left\{\alpha_{n}^{\prime}\right\}$, 
and $\left\{\alpha_{n}^{\prime \prime}\right\}$ be real number sequences in $(0,1)$. Let $\left\{r_{n}\right\}$ and $\left\{s_{n}\right\}$ be two positive real number sequences. Let $\left\{x_{n}\right\}$ be a sequence generated in the following process:

$$
\left\{\begin{array}{l}
x_{1} \in C, \\
F\left(z_{n}, z\right)+\left\langle A x_{n}, z-z_{n}\right\rangle+\frac{1}{r_{n}}\left\langle z-z_{n}, z_{n}-x_{n}\right\rangle \geq 0, \quad \forall z \in C, \\
y_{n}=P_{C}\left(z_{n}-s_{n} B z_{n}\right), \\
x_{n+1}=\alpha_{n} x_{n}+\alpha_{n}^{\prime} S^{n} y_{n}+\alpha_{n}^{\prime \prime} e_{n},
\end{array}\right.
$$

where $\left\{e_{n}\right\}$ is a bounded sequence in C. Assume that the control sequences satisfy the following restrictions:

(a) $\alpha_{n}+\alpha_{n}^{\prime}+\alpha_{n}^{\prime \prime}=1$

(b) $0<p \leq \alpha_{n} \leq p<1$ and $\sum_{n=1}^{\infty} \alpha_{n}^{\prime \prime}<\infty$;

(c) $0<s \leq s_{n} \leq s^{\prime}<2 \beta$ and $0<r \leq r_{n} \leq r^{\prime}<2 \alpha$,

where $p, q, s, s^{\prime}, r, r^{\prime}$ are real constants. Then $\left\{x_{n}\right\}$ converges weakly to some point in $\Omega$.

Further, if $S$ is an identity mapping, we have the following result.

Corollary 3.4 Let $C$ be a nonempty closed convex subset of a real Hilbert space $H$. Let $F$ be a bifunction from $C \times C$ to $\mathbb{R}$ which satisfies (A1)-(A4). Let $A: C \rightarrow H$ be an $\alpha$-inversestrongly monotone mapping, and let $B: C \rightarrow H$ be a $\beta$-inverse-strongly monotone mapping. Assume that $\Omega=\operatorname{VI}(C, B) \cap \operatorname{EP}(F, A)$ is not empty. Let $\left\{\alpha_{n}\right\},\left\{\alpha_{n}^{\prime}\right\}$, and $\left\{\alpha_{n}^{\prime \prime}\right\}$ be real number sequences in $(0,1)$. Let $\left\{r_{n}\right\}$ and $\left\{s_{n}\right\}$ be two positive real number sequences. Let $\left\{x_{n}\right\}$ be a sequence generated in the following process:

$$
\left\{\begin{array}{l}
x_{1} \in C \\
F\left(y_{n}, z\right)+\left\langle A x_{n}, z-y_{n}\right\rangle+\frac{1}{r_{n}}\left\langle z-y_{n}, y_{n}-x_{n}\right\rangle \geq 0 \\
x_{n+1}=\alpha_{n} x_{n}+\alpha_{n}^{\prime} P_{C}\left(y_{n}-s_{n} B y_{n}\right)+\alpha_{n}^{\prime \prime} e_{n},
\end{array}\right.
$$

where $\left\{e_{n}\right\}$ is a bounded sequence in C. Assume that the control sequences satisfy the following restrictions:

(a) $\alpha_{n}+\alpha_{n}^{\prime}+\alpha_{n}^{\prime \prime}=1$

(b) $0<p \leq \alpha_{n} \leq q<1$ and $\sum_{n=1}^{\infty} \alpha_{n}^{\prime \prime}<\infty$;

(c) $0<s \leq s_{n} \leq s^{\prime}<2 \beta$ and $0<r \leq r_{n} \leq r^{\prime}<2 \alpha$,

where $p, q, s, s^{\prime}, r, r^{\prime}$ are real constants. Then $\left\{x_{n}\right\}$ converges weakly to some point in $\Omega$.

Next, we give a result on variational inequality (2.1).

Corollary 3.5 Let $C$ be a nonempty closed convex subset of a real Hilbert space $H$. Let $A: C \rightarrow H$ be an $\alpha$-inverse-strongly monotone mapping, and let $B: C \rightarrow H$ be a $\beta$-inversestrongly monotone mapping. Assume that $\Omega=\operatorname{VI}(C, B) \cap \operatorname{VI}(C, A)$ is not empty. Let $\left\{\alpha_{n}\right\}$, $\left\{\alpha_{n}^{\prime}\right\}$, and $\left\{\alpha_{n}^{\prime \prime}\right\}$ be real number sequences in $(0,1)$. Let $\left\{r_{n}\right\}$ and $\left\{s_{n}\right\}$ be two positive real number sequences. Let $\left\{x_{n}\right\}$ be a sequence generated in the following process:

$$
\left\{\begin{array}{l}
x_{1} \in C \\
z_{n}=P_{C}\left(x_{n}-s_{n} A x_{n}\right) \\
x_{n+1}=\alpha_{n} x_{n}+\alpha_{n}^{\prime} P_{C}\left(z_{n}-s_{n} B z_{n}\right)+\alpha_{n}^{\prime \prime} e_{n}
\end{array}\right.
$$


where $\left\{e_{n}\right\}$ is a bounded sequence in C. Assume that the control sequences satisfy the following restrictions:

(a) $\alpha_{n}+\alpha_{n}^{\prime}+\alpha_{n}^{\prime \prime}=1$

(b) $0<p \leq \alpha_{n} \leq q<1$ and $\sum_{n=1}^{\infty} \alpha_{n}^{\prime \prime}<\infty$;

(c) $0<s \leq s_{n} \leq s^{\prime}<2 \beta$ and $0<r \leq r_{n} \leq r^{\prime}<2 \alpha$,

where $p, q, s, s^{\prime}, r, r^{\prime}$ are real constants. Then $\left\{x_{n}\right\}$ converges weakly to some point in $\Omega$.

Proof Putting $F \equiv 0$, we see that

$$
\left\langle A x_{n}, z-z_{n}\right\rangle+\frac{1}{r_{n}}\left\langle z-z_{n}, z_{n}-x_{n}\right\rangle \geq 0, \quad \forall z \in C
$$

is equivalent to

$$
\left\langle x_{n}-r_{n} A x_{n}-z_{n}, z_{n}-z\right\rangle \geq 0, \quad \forall z \in C .
$$

This implies that $z_{n}=P_{C}\left(x_{n}-r_{n} A x_{n}\right)$. Let $\beta_{n}=0$ and $S$ be the identity. Then we can obtain from Theorem 3.1 the desired results immediately.

Finally, we consider solving common fixed points of a pair of strict pseudocontractions.

Corollary 3.6 Let $C$ be a nonempty closed convex subset of a real Hilbert space H. Let $T_{1}: C \rightarrow C$ be an $\alpha$-strict pseudocontraction, and let $T_{2}: C \rightarrow C$ be a $\beta$-strict pseudocontraction. Assume that $\Omega=F\left(T_{1}\right) \cap F\left(T_{2}\right)$ is not empty. Let $\left\{\alpha_{n}\right\},\left\{\alpha_{n}^{\prime}\right\}$, and $\left\{\alpha_{n}^{\prime \prime}\right\}$ be real number sequences in $(0,1)$. Let $\left\{r_{n}\right\}$ and $\left\{s_{n}\right\}$ be two positive real number sequences. Let $\left\{x_{n}\right\}$ be a sequence generated in the following process:

$$
\left\{\begin{array}{l}
x_{1} \in C, \\
z_{n}=\left(1-r_{n}\right) x_{n}+r_{n} T_{2} x_{n}, \\
y_{n}=\left(1-s_{n}\right) x_{n}+s_{n} T_{1} x_{n}, \\
x_{n+1}=\alpha_{n} x_{n}+\alpha_{n}^{\prime} y_{n}+\alpha_{n}^{\prime \prime} e_{n},
\end{array}\right.
$$

where $\left\{e_{n}\right\}$ is a bounded sequence in C. Assume that the control sequences satisfy the following restrictions:

(a) $\alpha_{n}+\alpha_{n}^{\prime}+\alpha_{n}^{\prime \prime}=1$

(b) $0<p \leq \alpha_{n} \leq q<1$ and $\sum_{n=1}^{\infty} \alpha_{n}^{\prime \prime}<\infty$;

(c) $0<s \leq s_{n} \leq s^{\prime}<1-\alpha$ and $0<r \leq r_{n} \leq r^{\prime}<1-\beta$,

where $p, q, s, s^{\prime}, r, r^{\prime}$ are real constants. Then $\left\{x_{n}\right\}$ converges weakly to some point in $\Omega$.

Proof Put $F \equiv 0, A=I-T_{2}$ and $B=I-T_{1}$. It follows that $A$ is $\frac{1-\alpha}{2}$-inverse-strongly monotone and $B$ is $\frac{1-\beta}{2}$-inverse-strongly monotone. We also have $F\left(T_{1}\right)=\operatorname{VI}(C, B)$ and $F\left(T_{2}\right)=\mathrm{VI}(C, A)$. In view of Theorem 3.1, we find the desired result immediately. 


\section{References}

1. Facchinei, F, Pang, JS: Finite-Dimensional Variational Inequalities and Complementarity Problems, vol. I. Springer, New York (2003)

2. Facchinei, F, Pang, JS: Finite-Dimensional Variational Inequalities and Complementarity Problems, vol. II. Springer, New York (2003)

3. Fattorini, HO: Infinite-Dimensional Optimization and Control Theory. Cambridge University Press, Cambridge (1999)

4. Rockfellar, RT: Monotone operators and proximal point algorithm. SIAM J. Control Optim. 14, 877-898 (1976)

5. Rockafellar, RT: Convex Analysis. Princeton University Press, Princeton (1970)

6. Rockafellar, RT: On the maximality of sums of nonlinear monotone operators. Trans. Am. Math. Soc. 149, 75-88 (1970)

7. Tanaka, Y: A constructive version of Ky Fan's coincidence theorem. J. Math. Comput. Sci. 2, 926-936 (2012)

8. He, RH: Coincidence theorem and existence theorems of solutions for a system of Ky Fan type minimax inequalities in FC-spaces. Adv. Fixed Point Theory 2, 47-57 (2012)

9. Byrne, C: A unified treatment of some iterative algorithms in signal processing and image reconstruction. Inverse Probl. 20, 103-120 (2008)

10. Genel, A, Lindenstruss, J: An example concerning fixed points. Isr. J. Math. 22, 81-86 (1975)

11. Cho, SY, Qin, X, Kang, SM: Iterative processes for common fixed points of two different families of mappings with applications. J. Glob. Optim. 57, 1429-1446 (2013)

12. Takahashi, W, Toyoda, M: Weak convergence theorems for nonexpansive mappings and monotone mappings. J. Optim. Theory Appl. 118, 417-428 (2003)

13. Cho, YJ, Qin, X: Systems of generalized nonlinear variational inequalities and its projection methods. Nonlinear Anal. 69, 4443-4451 (2008)

14. LV, S, Wu, C: Convergence of iterative algorithms for a generalized variational inequality and a nonexpansive mapping. Eng. Math. Lett. 1, 44-57 (2012)

15. Chang, SS, Lee, HWJ, Chan, CK: A new hybrid method for solving a generalized equilibrium problem, solving a variational inequality problem and obtaining common fixed points in Banach spaces, with applications. Nonlinear Anal. 73, 2260-2270 (2010)

16. Cho, SY, Kang, SM: Approximation of fixed points of pseudocontraction semigroups based on a viscosity iterative process. Appl. Math. Lett. 24, 224-228 (2011)

17. Hao, Y: On variational inclusion and common fixed point problems in Hilbert spaces with applications. Appl. Math. Comput. 217, 3000-3010 (2010)

18. Yuan, Q, Kim, JK: Weak convergence of algorithms for asymptotically strict pseudocontractions in the intermediate sense and equilibrium problems. Fixed Point Theory Appl. 2012, 132 (2012)

19. Cho, SY, Kang, SM: Approximation of common solutions of variational inequalities via strict pseudocontractions. Acta Math. Sci. 32, 1607-1618(2012)

20. Luo, H, Wang, Y: Iterative approximation for the common solutions of a infinite variational inequality system for inverse-strongly accretive mappings. J. Math. Comput. Sci. 2, 1660-1670 (2012)

21. Cho, SY, Li, W, Kang, SM: Convergence analysis of an iterative algorithm for monotone operators. J. Inequal. Appl. 2013, 199 (2013)

22. Zegeye, H, Shahzad, N: Strong convergence theorem for a common point of solution of variational inequality and fixed point problem. Adv. Fixed Point Theory 2, 374-397 (2012)

23. Browder, FE, Petryshyn, WV: Construction of fixed points of nonlinear mappings in Hilbert space. J. Math. Anal. Appl. 20, 197-228 (1967)

24. Qihou, L: Convergence theorems of the sequence of iterates for asymptotically demicontractive and hemicontractive mappings. Nonlinear Anal. 26, 1835-1845 (1996)

25. Kim, KS, Kim, JK, Lim, WH: Convergence theorems for common solutions of various problems with nonlinear mapping. J. Inequal. Appl. 2014, 2 (2014)

26. Qin, X, Chang, SS, Cho, YJ: Iterative methods for generalized equilibrium problems and fixed point problems with applications. Nonlinear Anal. 11, 2963-2972 (2010)

27. Rodjanadid, B, Sompong, S: A new iterative method for solving a system of generalized equilibrium problems, generalized mixed equilibrium problems and common fixed point problems in Hilbert spaces. Adv. Fixed Point Theory 3, 675-705 (2013)

28. Kang, SM, Cho, SY, Liu, Z: Convergence of iterative sequences for generalized equilibrium problems involving inverse-strongly monotone mappings. J. Inequal. Appl. 2010, Article ID 827082 (2010)

29. Chang, SS, Zuo, P: Shrinking projection method of common solutions for generalized equilibrium quasi- $\phi$-nonexpansive mapping and relatively nonexpansive mapping. J. Inequal. Appl. 2010, Article ID 101690 (2010)

30. Zhang, Q, Wu, H: Hybrid algorithms for equilibrium and common fixed point problems with applications. J. Inequal. Appl. 2014, $221(2014)$

31. Cho, SY, Qin, X: On the strong convergence of an iterative process for asymptotically strict pseudocontractions and equilibrium problems. Appl. Math. Comput. 235, 430-438 (2014)

32. Blum, E, Oettli, W: From optimization and variational inequalities to equilibrium problems. Math. Stud. 63, 123-145 (1994)

33. Schu, J: Weak and strong convergence to fixed points of asymptotically nonexpansive mappings. Bull. Aust. Math. Soc. 43, 153-159 (1991)

34. Tan, KK, Xu, HK: Approximating fixed points of nonexpansive mappings by the Ishikawa iterative process. J. Math. Anal. Appl. 178, 301-308 (1993)

35. Opial, Z: Weak convergence of the sequence of successive approximation for nonexpansive mappings. Bull. Am. Math. Soc. 73, 591-597 (1967)

10.1186/1029-242X-2014-378

Cite this article as: Hao: Weak convergence theorems of a hybrid algorithm in Hilbert spaces. Journal of Inequalities and Applications 2014, 2014:378 